\title{
Erratum to: Studying high impact fix-inducing changes
}

\author{
Ayse $\operatorname{Tosun}^{1} \cdot$ Emad Shihab $^{2} \cdot$ Yasutaka Kamei $^{3}$
}

Published online: 1 September 2016

(C) Springer Science+Business Media New York 2016

\section{Erratum to: Empir Software Eng (2016) 21:605-641 \\ DOI 10.1007/s10664-015-9370-z}

The original version of this article unfortunately contained a mistake. The name of the third author was incorrectly displayed as "Yasukata Kamei". The correct information is as shown above.

The online version of the original article can be found at http://dx.doi.org/10.1007/s10664-015-9370-z.

Ayse Tosun

tosunay@itu.edu.tr

Emad Shihab

eshihab@cse.concordia.ca

Yasutaka Kamei

kamei@ait.kyushu-u.ac.jp

1 Faculty of Computer and Informatics, Istanbul Technical University, Istanbul, Turkey

2 Department of Computer Science and Software Engineering, Concordia University, Quebec, Canada

3 Graduate School and Faculty of Information Science and Electrical Engineering, Kyushu University, Fukuoka, Japan 